\title{
A Monte Carlo Study of the Second Virial Coefficient of Semiflexible Regular Three-Arm Star Polymers
}

\author{
By Daichi IDA and Takenao YOSHIZAKI*
}

\begin{abstract}
A Monte Carlo (MC) study is made of the second virial coefficient $A_{2}$ along with the mean-square radius of gyration $\left\langle S^{2}\right\rangle$ for regular three-arm star and linear freely rotating chains of bond angles $\theta$ ranging from $109^{\circ}$ (typical flexible chain) to $175^{\circ}$ (typical semiflexible or stiff chain) with the Lennard-Jones 6-12 potentials between beads corresponding to a good solvent system, in the range of the total number $n$ of bonds from 30 to 900 . On the basis of the MC values of $A_{2}$ so obtained, an examination is made of effects of chain stiffness on the ratio $g_{A_{2}}$ of $A_{2}$ of the star chain to that of the linear one, both chains having the same $n$ and $\theta$. It is then found that $g_{A_{2}}$ is rather insensitive to change in $\theta$ (chain stiffness) in contrast to the cases of the ratios $g_{S}$ and $g_{\eta}$ related to $\left\langle S^{2}\right\rangle$ and the intrinsic viscosity $[\eta]$, respectively, which remarkably decrease with increasing $\theta$. KEY WORDS: Regular Three-Arm Star Polymer / Semiflexible Polymer / Second Virial Coefficient / Monte Carlo Simulation / Freely Rotating Chain /
\end{abstract}

In previous papers, ${ }^{1,2}$ we have examined effects of chain stiffness on the intrinsic viscosity $[\eta]$ of unperturbed regular three-arm star polymer chains by Monte Carlo (MC) simulations $^{1}$ or on the basis of the Kirkwood-Riseman theory. ${ }^{2-4}$ The ratio $g_{\eta}$ of $[\eta]$ of an unperturbed regular three-arm star chain to that of an unperturbed linear one, both having the same molecular weight and chain stiffness, has been shown to become remarkably smaller than the value $c a$. 0.9 in the random-coil limit ${ }^{5,6}$ as the chains become stiffer, as in the case of the ratio $g_{S}$ of the mean-square radius of gyration $\left\langle S^{2}\right\rangle$ of the former chain to that of the latter. ${ }^{7}$ We note that $g_{S}$ becomes $7 / 9$ in the random-coil limit. ${ }^{8}$ Since $[\eta]$ and $\left\langle S^{2}\right\rangle$ are measures of the average chain dimension, the above-mentioned results for $g_{\eta}$ and $g_{S}$ indicate that the average dimension of the unperturbed regular three-arm star chain becomes much smaller than that of the corresponding linear one as the chains become stiffer.

In good solvents (perturbed state), short-ranged repulsive interactions work between segments constituting the polymer chains, expanding the individual chains and yielding an effective volume $V_{\mathrm{E}}$ excluded to one chain by the presence of another. The volume $V_{\mathrm{E}}$ is another measure of the average chain dimension, although well-defined only in a good solvent system or in the perturbed state. The quantity $V_{\mathrm{E}}$ may be defined from the second virial coefficient $A_{2}$ as follows, ${ }^{4}$

$$
A_{2}=4 N_{\mathrm{A}} V_{\mathrm{E}} / M^{2}
$$

where $N_{\mathrm{A}}$ is the Avogadro constant and $M$ is the molecular weight. It is then interesting to examine the effects of chain stiffness on the ratio $g_{A_{2}}$ of $A_{2}$ of a regular three-arm star chain to that of the corresponding linear one. In this paper, we make an MC study of $g_{A_{2}}$ as a necessary continuation of the previous papers. ${ }^{1,2}$
An available theoretical value of $g_{A_{2}}$ for the regular threearm star chain is 0.968 in the random-coil limit obtained by Douglas and Freed ${ }^{9}$ on the basis of the polymer renormalization group (RG) theory, being nearly equal to unity. Then the main question we must answer in this paper is whether or not $g_{A_{2}}$ becomes remarkably smaller than unity as the chains become stiffer, as in the cases of $g_{\eta}$ and $g_{S}$.

\section{MODELS AND METHODS}

The MC models used in this study are the same as those used in the previous one ${ }^{1}$ except for the value of a parameter of interactions between segments (beads).

The star chain model is the regular three-arm star freely rotating chain, each arm composed of $m$ successive bonds of length unity, so that it is composed of $3 m(=n)$ bonds, in total, and $3 m+1$ beads whose centers are located at $3 m-3$ junctions of two successive bonds on the arms, at the three terminal ends, and at the branch point (center) (see Figure 1 of ref 1). The angle between each pair of the bonds connected to the center is fixed to be $120^{\circ}$, so that those bonds are on the same plane. For convenience, the three arms are designated the first, second, and third ones and the beads on the $i$ th arm $(i=1,2,3)$ are numbered $(i-1) m+1,(i-1) m+2, \cdots, i m$ from the center to the end, with the center bead numbered 0 . The $i$ th bond vector $(1 \leq i \leq 3 m ; i \neq m+1,2 m+1)$ connects the centers of the $(i-1)$ th and $i$ th beads with its direction from the $(i-1)$ th to $i$ th bead, and the $(m+1)$ th and $(2 m+1)$ th bond vectors are from the 0 th to the $(m+1)$ th and $(2 m+1)$ th beads, respectively. All the $3 m-3$ bond angles $\theta$ (not supplement) except for those around the center are fixed, so that the configuration of the entire chain may be specified by the set of $3 m-3$ rotation angles $\left\{\phi_{3 m-3}\right\}=\left(\phi_{1}, \cdots, \phi_{m-1}, \phi_{m+1}, \cdots\right.$, $\left.\phi_{2 m-1}, \phi_{2 m+1}, \cdots, \phi_{3 m-1}\right)$ apart from its position and orientation 


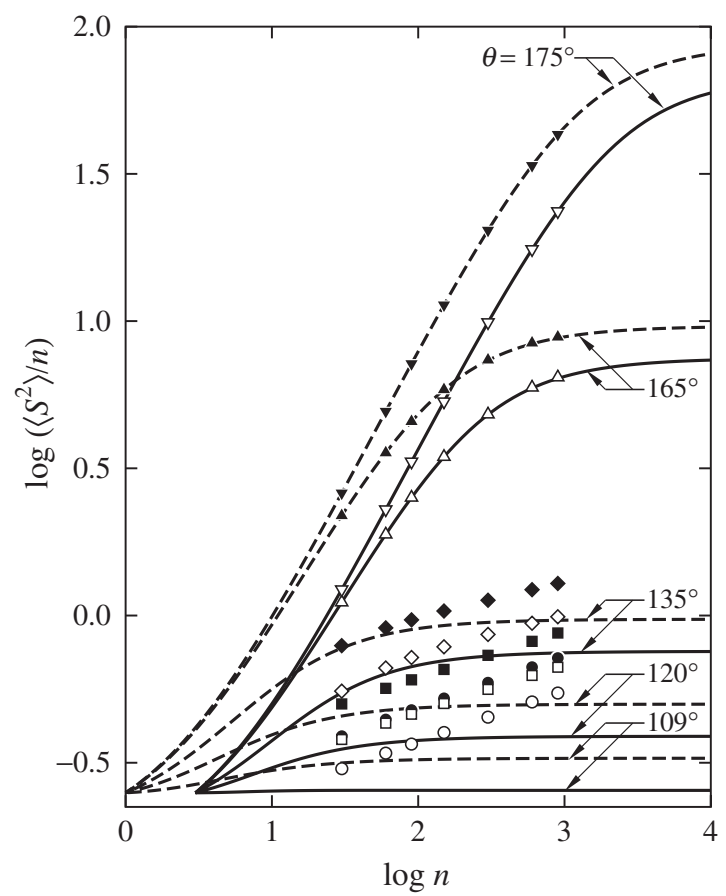

Figure 1. Double-logarithmic plots of $\left\langle S^{2}\right\rangle / n$ against $n$ at $T^{*}=8.0(\mathrm{good}$ solvent system). The open and closed symbols represent the $\mathrm{MC}$ values for the regular three-arm star and linear freely rotating chains, respectively, of $\theta=109^{\circ}(\bigcirc, \bigcirc), 120^{\circ}(\square, \square), 135^{\circ}(\diamond$, $\diamond), 165^{\circ}(\triangle, \boldsymbol{\Delta})$, and $175^{\circ}(\nabla, \nabla)$. The solid and dashed curves represent the theoretical values for the ideal regular three-arm star and linear freely rotating chains, respectively, with the indicated values of $\boldsymbol{\theta}$.

in an external Cartesian coordinate system, where $\phi_{i}$ is the internal rotation angle around the $i$ th bond vector.

The linear chain model, the counterpart of the above star one, is the freely rotating chain composed of $n$ bonds of length unity and $n+1$ beads, whose centers are located at the $n-1$ junctions of two successive bonds and at the two terminal ends. We set $n$ equal to $3 m$. The beads are numbered $0,1,2, \cdots, n$ from one end to the other, and the $i$ th bond vector $(i=$ $1,2, \cdots, n)$ connects the centers of the $(i-1)$ th and $i$ th beads with its direction from the $(i-1)$ th to the $i$ th bead. All the $n-1$ bond angles are fixed at $\theta$, so that the configuration of the linear chain may be specified by the set of $n-2$ internal rotation angles $\left\{\phi_{n-2}\right\}=\left(\phi_{2}, \phi_{3}, \cdots, \phi_{n-1}\right)$ apart from its position and orientation in the external Cartesian coordinate system.

The total potential energy $U$ of the regular three-arm star chain as a function of $\left\{\phi_{3 m-3}\right\}$ may be given by

$$
\begin{aligned}
U\left(\left\{\phi_{3 m-3}\right\}\right)= & \sum_{i=0}^{1} \sum_{j=i+1}^{2} \sum_{k, l=1}^{m} h(k+l-4) u\left(R_{(i m+k)(j m+l)}\right) \\
& +\sum_{i=0}^{2} \sum_{j=1}^{m-4} \sum_{k=j+4}^{m} u\left(R_{(i m+j)(i m+k)}\right) \\
& +\sum_{i=0}^{2} \sum_{j=4}^{m} u\left(R_{0(i m+j)}\right)
\end{aligned}
$$

(regular three-arm star)

and that of the linear one as a function of $\left\{\phi_{n-2}\right\}$ by

$$
U\left(\left\{\phi_{n-2}\right\}\right)=\sum_{i=0}^{n-4} \sum_{j=i+4}^{n} u\left(R_{i j}\right) \quad \text { (linear) }
$$

where $h(x)$ is a unit step function such that $h(x)=1$ for $x \geq 0$ and $h(x)=0$ for $x<0$ and $R_{i j}$ is the distance between the centers of the $i$ th and $j$ th beads. We note that in eqs 2 and 3 the interactions between the third-neighbor beads along the chain have been neglected, since they seem to make the chain locally take the cis conformation to excess.

Similarly, the total intermolecular (excluded-volume) potential energy $U_{12}(1,2)$ between two chains 1 and 2 as a function of all the coordinates of chains 1 and 2 may be given by

$$
U_{12}(1,2)=\sum_{i_{1}=0}^{n} \sum_{i_{2}=0}^{n} u\left(R_{i_{1} i_{2}}\right)
$$

where $R_{i_{1} i_{2}}$ is the distance between the centers of the $i_{1}$ th bead of chain 1 and of the $i_{2}$ th one of chain 2 . We note that we use the McMillan-Mayer symbolism ${ }^{4,10}$ to formulate $A_{2}$, here and hereafter. Then the $i$ th bead $(i=0,1,2, \cdots, n)$ of chain $\alpha$ $(\alpha=1,2)$ is labeled as $i_{\alpha}$, and the symbol $(\alpha)(\alpha=1,2)$ denotes all the coordinates (external and internal) of chain $\alpha$.

We adopt as the pair potential $u(R)$ (of mean force) the cutoff version of the Lennard-Jones (LJ) 6-12 potential given by

$$
\begin{aligned}
u(R) & =\infty & & \text { for } 0 \leq R<c \sigma \\
& =u^{\mathrm{LJ}}(R) & & \text { for } c \sigma \leq R<3 \sigma \\
& =0 & & \text { for } 3 \sigma \leq R
\end{aligned}
$$

where $u^{\mathrm{LJ}}(R)$ is the $\mathrm{LJ}$ potential ${ }^{11}$ given by

$$
u^{\mathrm{LJ}}(R)=4 \epsilon\left[\left(\frac{\sigma}{R}\right)^{12}-\left(\frac{\sigma}{R}\right)^{6}\right]
$$

with $\sigma$ and $\epsilon$ the collision diameter and the depth of the potential well at the minimum of $u^{\mathrm{LJ}}(R)$, respectively. We note that $u(R)$ given by eq 5 is the LJ potential cut off at the upper bound $3 \sigma$. The lower bound $c \sigma$ in eq 5 has been introduced for numerical convenience; the factor $c$ is properly chosen so that the Boltzmann factor $e^{-u^{\mathrm{LJ}} / k_{\mathrm{B}} T}$ may be regarded as numerically vanishing compared to unity, where $k_{\mathrm{B}}$ is the Boltzmann constant and $T$ is the absolute temperature. In practice, in double-precision computation, we put

$$
c=\left[2 /\left(1+\sqrt{1+36 T^{*}}\right)\right]^{1 / 6}
$$

so that $e^{-u^{\mathrm{LJ}} / k_{\mathrm{B}} T} \lesssim 2 \times 10^{-16}$ for $0 \leq R<c \sigma$, where $T^{*}$ is the reduced temperature defined by $T^{*}=k_{\mathrm{B}} T / \epsilon$. Further, we put $\sigma=1$ for simplicity, as previously ${ }^{1}$ done. We note that the value 3.72 of $T^{*}$ corresponds to the $\Theta$ temperature and 8.0 to a good solvent system. ${ }^{12,13}$

The mean-square radius of gyration $\left\langle S^{2}\right\rangle$, i.e., the ensemble average of the square radius of gyration $S^{2}$ may be evaluated from

$$
\left\langle S^{2}\right\rangle=N_{\mathrm{s}}^{-1} \sum_{\left\{\phi_{k}\right\}} S^{2}\left(\left\{\phi_{k}\right\}\right)
$$

where the sum is taken over $N_{\mathrm{s}}$ sample configurations $\left\{\phi_{k}\right\}$ with $k$ being equal to $3 m-3$ for the star chain and $n-2$ for the 
linear one, $\left\{\phi_{k}\right\}$ generated in an MC run by an application of the pivot algorithm ${ }^{14,15}$ and the Metropolis method of importance sampling ${ }^{16}$ as done in the previous MC studies. ${ }^{1,12}$ For each sample configuration, $S^{2}$ as a function of $\left\{\phi_{k}\right\}$ may be calculated from

$$
S^{2}=\frac{1}{n+1} \sum_{i=0}^{n}\left|\mathbf{r}_{i}-\mathbf{r}_{\mathrm{c} . \mathrm{m} .}\right|^{2}
$$

with $\mathbf{r}_{i}$ the vector position of the center of the $i$ th bead and $\mathbf{r}_{\text {c.m. }}$ the vector position of the center of mass of the chain given by

$$
\mathbf{r}_{\mathrm{c} . \mathrm{m} .}=\frac{1}{n+1} \sum_{i=0}^{n} \mathbf{r}_{i}
$$

In every MC run, an initial configuration is generated by trial and error so that all the distances between the centers of beads are greater than or equal to $c$. (Note that $\sigma=1$.) One configuration is sampled at every $M_{\text {nom }}$ (nominal) pivot steps, so that $N_{\mathrm{s}} \times M_{\text {nom }}$ pivot steps are required to obtain a set of $N_{\mathrm{s}}$ sample configurations. The number of $M_{\text {nom }}$ has been chosen to be $c a$. $10 n$ for the regular three-arm star chains and $c a$. $2 n$ for the linear ones.

As for the second virial coefficient $A_{2}$, it may be evaluated from

$$
A_{2}=\frac{2 \pi N_{\mathrm{A}}}{M^{2}} \int_{0}^{\infty}\left\{1-\exp \left[-\frac{\bar{U}_{12}(r)}{k_{\mathrm{B}} T}\right]\right\} r^{2} d r
$$

where $\bar{U}_{12}(r)$ is the averaged intermolecular potential as a function of the distance $r=|\mathbf{r}|$ between the centers of mass of chains 1 and 2 defined by

$$
\bar{U}_{12}(r)=-k_{\mathrm{B}} T \ln \left\langle\exp \left[-\frac{U_{12}(1,2)}{k_{\mathrm{B}} T}\right]\right\rangle_{r}
$$

In eq $12,\langle\cdots\rangle_{r}$ indicates the conditional equilibrium average taken over the configurations of the two chains with $\mathbf{r}$ fixed by the use of the single-chain distribution function for each with the intramolecular excluded-volume potential for the star and linear chains given by eqs 2 and 3 , respectively. In practice, the conditional average may be calculated by the use of a set of $N_{\mathrm{s}}$ sample configurations $\left\{\phi_{k}\right\}$ generated above, as follows. First, we randomly sample a pair of sample configurations (chains 1 and 2) from the set and calculate the intermolecular potential $U_{12}$ from eq 4 at given $r$ after randomizing the orientations of the two configurations in the external coordinate system. Numerical evaluation of $U_{12}$ may be carried out following the procedure used in the previous study ${ }^{13}$ of $A_{2}$ with the use of the "zippering" method. ${ }^{17,18}$ Then the average on the right-hand side of eq 12 may be evaluated from

$$
\left\langle\exp \left[-\frac{U_{12}(1,2)}{k_{\mathrm{B}} T}\right]\right\rangle_{r}=N_{\mathrm{p}}^{-1} \sum_{(1,2)}^{\prime} \exp \left[-\frac{U_{12}(1,2)}{k_{\mathrm{B}} T}\right]
$$

where $\sum_{(1,2)}^{\prime}$ indicates summation over $N_{\mathrm{p}}$ pairs of sample configurations $(1,2)$ at given $r$. With the values of $\bar{U}_{12}(r)$ so evaluated for various values of $r$ at given $T^{*}$, the quantity $A_{2} M^{2}$ for given $n$ may then be calculated from eq 11 by numerical integration with the use of the trapezoidal rule formula.
In the practical evaluation of $\left\langle S^{2}\right\rangle$ and $A_{2}$ for the regular three-arm star and linear freely rotating chains at $T^{*}=8.0$ (good solvent system), we have generated 10 sets of $10^{5}\left(=N_{\mathrm{s}}\right)$ sample configurations for $n=30,60,90,150$, and 300, 5 sets of those for $n=600$, and 2 sets of those for $n=900$. In the evaluation of $\bar{U}_{12}$ (and $\left.A_{2}\right), 10^{6}$ or $10^{7}\left(=N_{\mathrm{p}}\right)$ sample pairs have been taken from each set. Then the total number of sample pairs is equal to the number $N_{\mathrm{p}}$ of sample pairs in each set multiplied by the number of sets.

All the numerical work has been done by the use of a personal computer with an Intel Core2 Duo E6600 CPU. A source program coded in $\mathrm{C}$ has been compiled by the GNU $\mathrm{C}$ compiler version 3.4.6 with real variables of double precision. For a generation of pseudorandom numbers, the subroutine package MT19937 supplied by Matsumoto and Nishimura ${ }^{19}$ has been used instead of the subroutine RAND included in the standard C library.

\section{RESULTS AND DISCUSSION}

\section{Mean-Square Radius of Gyration}

We have carried out MC runs to generate sample configurations for the regular three-arm star and linear freely rotating chains with $n(=3 m)=30,60,90,150,300,600$, and 900 and $\theta=109^{\circ}, 120^{\circ}, 135^{\circ}, 165^{\circ}$, and $175^{\circ}$ at $T^{*}=8.0(\operatorname{good}$ solvent system). The chain of $\theta=109^{\circ}$ corresponds to a typical flexible chain, and the chain becomes stiffer with increasing $\theta$ from $109^{\circ}$ to $175^{\circ}$.

The MC values of $\left\langle S^{2}\right\rangle / n$ for the star and linear chains are given in the second and fourth columns, respectively, of Table I, the number in the parentheses attached to each value indicating its statistical error. The $\left\langle S^{2}\right\rangle / n$ value and its error for each chain are the mean and the standard deviation, respectively, of independent $\mathrm{MC}$ results.

Figure 1 shows double-logarithmic plots of $\left\langle S^{2}\right\rangle / n$ against $n$ at $T^{*}=8.0($ good solvent system). The open and closed symbols represent the MC values for the star and linear chains, respectively, of $\theta=109^{\circ}(\bigcirc, \bigcirc), 120^{\circ}(\square, \boldsymbol{\square}), 135^{\circ}(\diamond, \diamond)$, $165^{\circ}(\triangle, \boldsymbol{\Delta})$, and $175^{\circ}(\nabla, \boldsymbol{\nabla})$. The solid and dashed curves represent the theoretical values of $\left\langle S^{2}\right\rangle / n$ for the ideal regular three-arm star and linear freely rotating chains, respectively, without interactions between beads, which have been calculated from

$$
\begin{aligned}
\left\langle S^{2}\right\rangle= & \frac{7}{54} \frac{1-\cos \theta}{1+\cos \theta} n+\frac{1}{54} \frac{31+90 \cos \theta-13 \cos ^{2} \theta}{(1+\cos \theta)^{2}} \\
& +\frac{1}{18} \frac{19+49 \cos \theta+71 \cos ^{2} \theta+5 \cos ^{3} \theta}{(1+\cos \theta)^{3}} \frac{1}{n+1} \\
& -\frac{2(1+2 \cos \theta)}{(1+\cos \theta)^{3}} \frac{1-(-\cos \theta)^{n / 3+1}}{n+1} \\
& +\frac{1}{27} \frac{(1-\cos \theta)\left(37-16 \cos \theta+\cos ^{2} \theta\right)}{(n+1)^{2}} \frac{1}{(1+\cos \theta)^{3}} \\
& -\frac{2\left(2+\cos ^{2} \theta\right)}{(1+\cos \theta)^{4}} \frac{1-(-\cos \theta)^{n / 3+1}}{(n+1)^{2}}
\end{aligned}
$$


Table I. Values of $\left\langle S^{2}\right\rangle / n$ and $A_{2} M_{\mathrm{b}}{ }^{2} / N_{\mathrm{A}}$ at $T^{*}=8.0$ (good solvent system)

\begin{tabular}{|c|c|c|c|c|}
\hline \multirow{2}{*}{$n$} & \multicolumn{2}{|c|}{ three-arm star } & \multicolumn{2}{|c|}{ linear } \\
\hline & $\left\langle S^{2}\right\rangle / n$ (error \%) & $A_{2} M_{\mathrm{b}}^{2} / N_{\mathrm{A}}$ (error \%) & $\left\langle S^{2}\right\rangle / n$ (error \%) & $A_{2} M_{\mathrm{b}}^{2} / N_{\mathrm{A}}($ error $\%)$ \\
\hline & \multicolumn{4}{|c|}{$\theta=109^{\circ}$} \\
\hline 30 & $0.302_{0}(0.1)$ & $0.251_{5}(0.1)$ & $0.389_{3}(0.1)$ & $0.261_{5}(0.2)$ \\
\hline 60 & $0.340_{7}(0.1)$ & $0.203_{4}(0.1)$ & $0.443_{3}(0.1)$ & $0.213_{4}(0.2)$ \\
\hline 90 & $0.366_{1}(0.1)$ & $0.181_{8}(0.1)$ & $0.477_{3}(0.1)$ & $0.191_{5}(0.1)$ \\
\hline 150 & $0.400_{4}(0.1)$ & $0.159_{0}(0.1)$ & $0.523_{4}(0.1)$ & $0.168_{4}(0.1)$ \\
\hline 300 & $0.451_{5}(0.1)$ & $0.133_{6}(0.1)$ & $0.591_{5}(0.1)$ & $0.142_{1}(0.1)$ \\
\hline 600 & $0.508_{8}(0.1)$ & $0.113_{1}(0.1)$ & $0.667_{7}(0.1)$ & $0.120_{3}(0.2)$ \\
\hline \multirow[t]{2}{*}{900} & $0.545_{8}(0.1)$ & $0.102_{5}(0.0)$ & $0.716_{9}(0.1)$ & $0.109_{5}(0.1)$ \\
\hline & \multicolumn{4}{|c|}{$\theta=120^{\circ}$} \\
\hline 30 & $0.379_{4}(0.1)$ & $0.2859(0.1)$ & $0.500_{9}(0.1)$ & $0.301_{1}(0.2)$ \\
\hline 60 & $0.431_{1}(0.1)$ & $0.241_{4}(0.1)$ & $0.565_{9}(0.1)$ & $0.252_{9}(0.2)$ \\
\hline 90 & $0.462_{2}(0.1)$ & $0.218_{9}(0.1)$ & $0.604_{8}(0.1)$ & $0.229_{8}(0.1)$ \\
\hline 150 & $0.502_{6}(0.1)$ & $0.194_{2}(0.1)$ & $0.656_{0}(0.1)$ & $0.205_{0}(0.1)$ \\
\hline 300 & $0.561_{3}(0.1)$ & $0.165_{9}(0.2)$ & $0.732_{6}(0.1)$ & $0.175_{7}(0.1)$ \\
\hline 600 & $0.626_{0}(0.1)$ & $0.141_{8}(0.1)$ & $0.817_{3}(0.1)$ & $0.150_{5}(0.2)$ \\
\hline \multirow[t]{2}{*}{900} & $0.667_{5}(0.0)$ & $0.129_{3}(0.1)$ & $0.872_{3}(0.0)$ & $0.137_{5}(0.0)$ \\
\hline & \multicolumn{4}{|c|}{$\theta=135^{\circ}$} \\
\hline 30 & $0.553_{7}(0.1)$ & $0.327_{4}(0.2)$ & $0.790_{1}(0.1)$ & $0.336_{2}(0.2)$ \\
\hline 60 & $0.663_{5}(0.1)$ & $0.287_{3}(0.3)$ & $0.9079(0.2)$ & $0.297_{3}(0.1)$ \\
\hline 90 & $0.720_{1}(0.1)$ & $0.268_{4}(0.1)$ & $0.966_{6}(0.2)$ & $0.278_{8}(0.2)$ \\
\hline 150 & $0.782_{9}(0.1)$ & $0.246_{3}(0.2)$ & $1.03_{6} \quad(0.1)$ & $0.256_{9}(0.2)$ \\
\hline 300 & $0.862_{6}(0.1)$ & $0.219_{0}(0.1)$ & $1.12_{7} \quad(0.1)$ & $0.229_{4}(0.1)$ \\
\hline 600 & $0.941_{4}(0.1)$ & $0.193_{5}(0.1)$ & $1.22_{4} \quad(0.1)$ & $0.203_{1}(0.1)$ \\
\hline \multirow[t]{2}{*}{900} & $0.990_{4}(0.0)$ & $0.179_{7}(0.0)$ & $1.28_{5} \quad(0.1)$ & $0.188_{7}(0.0)$ \\
\hline & \multicolumn{4}{|c|}{$\theta=165^{\circ}$} \\
\hline 30 & $1.10_{9} \quad(0.0)$ & $0.355_{6}(0.4)$ & $2.18_{5} \quad(0.0)$ & $0.352_{4}(0.4)$ \\
\hline 60 & $1.88_{6} \quad(0.0)$ & $0.330_{7}(0.3)$ & $3.57_{1} \quad(0.0)$ & $0.331_{4}(0.4)$ \\
\hline 90 & $2.51_{6} \quad(0.0)$ & $0.323_{3}(0.4)$ & $4.56_{1} \quad(0.1)$ & $0.323_{6}(0.6)$ \\
\hline 150 & $3.46_{1} \quad(0.0)$ & $0.316_{1}(0.3)$ & $5.85_{1} \quad(0.1)$ & $0.317_{7}(0.2)$ \\
\hline 300 & $4.82_{3} \quad(0.1)$ & $0.310_{0}(0.3)$ & $7.378 \quad(0.1)$ & $0.311_{6}(0.3)$ \\
\hline 600 & $5.95_{1} \quad(0.1)$ & $0.304_{4}(0.1)$ & $8.42_{8} \quad(0.1)$ & $0.307_{5}(0.1)$ \\
\hline \multirow[t]{2}{*}{900} & $6.44_{4} \quad(0.1)$ & $0.303_{0}(0.1)$ & $8.82_{9} \quad(0.0)$ & $0.305_{2}(0.3)$ \\
\hline & \multicolumn{4}{|c|}{$\theta=175^{\circ}$} \\
\hline 30 & $1.22_{6} \quad(0.0)$ & $0.354_{3}(0.3)$ & $2.60_{5} \quad(0.0)$ & $0.343_{8}(0.6)$ \\
\hline 60 & $2.29_{2} \quad(0.0)$ & $0.327_{9}(0.4)$ & $4.93_{6} \quad(0.0)$ & $0.320_{1}(0.8)$ \\
\hline 90 & $3.33_{0} \quad(0.0)$ & $0.319_{1}(0.5)$ & $7.16_{4} \quad(0.0)$ & $0.311_{8}(0.5)$ \\
\hline 150 & $5.32_{8} \quad(0.0)$ & $0.310_{9}(0.3)$ & $11.3_{4} \quad(0.0)$ & $0.307_{3}(0.8)$ \\
\hline 300 & $9.90_{0} \quad(0.0)$ & $0.304_{1}(0.5)$ & $20.3_{4}$ & $0.302_{9}(0.5)$ \\
\hline 600 & $17.5_{3} \quad(0.1)$ & $0.301_{8}(0.3)$ & $33.7_{4}$ & $0.299_{9}(0.9)$ \\
\hline 900 & $23.5_{9} \quad(0.1)$ & $0.299_{7}(0.5)$ & $43.0_{3} \quad(0.1)$ & $0.297_{0}(0.6)$ \\
\hline
\end{tabular}

$$
+\frac{3}{(1+\cos \theta)^{4}} \frac{\left[1-(-\cos \theta)^{n / 3+1}\right]^{2}}{(n+1)^{2}}
$$

(regular three-arm star)

and

$$
\begin{aligned}
\left\langle S^{2}\right\rangle= & \frac{1}{6} \frac{1-\cos \theta}{1+\cos \theta} n+\frac{1}{6} \frac{1+6 \cos \theta-\cos ^{2} \theta}{(1+\cos \theta)^{2}} \\
& +\frac{1-1-7 \cos \theta+7 \cos ^{2} \theta+\cos ^{3} \theta}{6} \frac{1}{(1+\cos \theta)^{3}} \\
& -\frac{2 \cos ^{2} \theta}{(1+\cos \theta)^{4}} \frac{1-(-\cos \theta)^{n+1}}{(n+1)^{2}} \quad \text { (linear) }
\end{aligned}
$$

respectively, with the indicated values of $\theta$. We note that eq 14 is a special case of the theoretical expression obtained by Guenza et al. ${ }^{20}$ for the regular $f$-arm star freely rotating chain.

In the cases of $\theta=109^{\circ}, 120^{\circ}$ and $135^{\circ}$, the MC values for both the star and linear chains deviate upward from the corresponding ideal-chain ones because of the intramolecular excluded-volume effect and also of the effect of the interactions between beads on the unperturbed chain dimension through the short-range interference. ${ }^{12,21,22}$ In the cases of $\theta=$ $165^{\circ}$ and $175^{\circ}$, on the other hand, the MC values for both the star and linear chains are almost identical with the corresponding ideal-chain values, because the effects of the intramolecular interactions between beads become negligibly small for a 


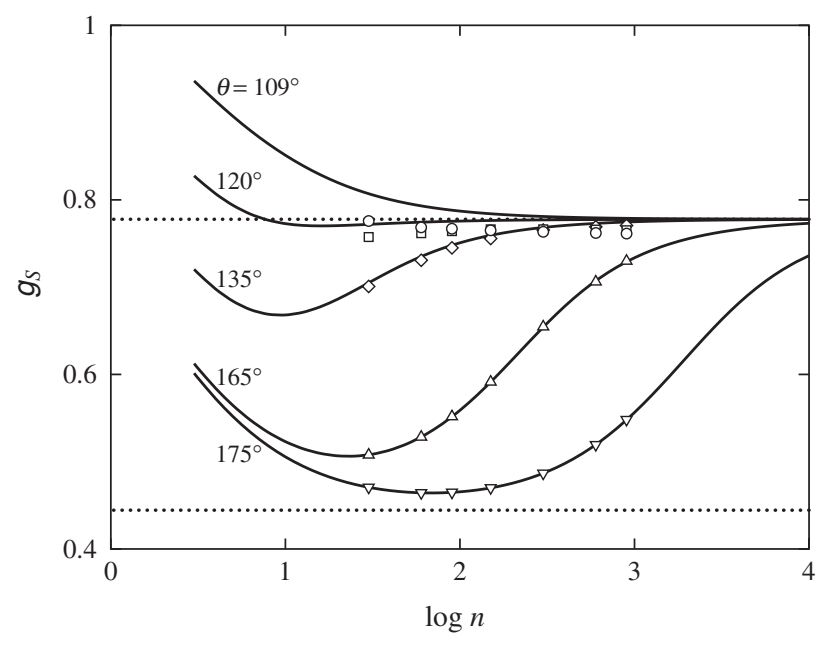

Figure 2. Plots of $g_{s}$ against $\log n$ at $T^{*}=8.0$ (good solvent system) for the freely rotating chains of $\theta=109^{\circ}(\bigcirc), 120^{\circ}(\square), 135^{\circ}(\diamond), 165^{\circ}$ $(\triangle)$, and $175^{\circ}(\nabla)$. The solid curves represent the theoretical values for the ideal freely rotating chains with the indicated values of $\boldsymbol{\theta}$. The upper and lower dotted horizontal lines represent the asymptotic values $7 / 9$ in the ideal random-coil limit and 4/9 in the rigid-rod limit, respectively.

typical stiff chain if its total contour length is not very long. We note that the ring-closure or intramolecular-contact probability approaches zero in the rigid-rod limit and therefore the intramolecular excluded-volume effect vanishes in the limit.

Figure 2 shows plots of $g_{S}$ against $\log n$ at $T^{*}=8.0(\operatorname{good}$ solvent system), where $g_{S}$ is the ratio of $\left\langle S^{2}\right\rangle$ of the regular three-arm star chain to that of the corresponding linear one. The symbols represent the MC values of $g_{S}$ calculated from the values of $\left\langle S^{2}\right\rangle / n$ given in the second and fourth columns in Table I for $\theta=109^{\circ}(\bigcirc), 120^{\circ}(\square), 135^{\circ}(\diamond), 165^{\circ}(\triangle)$, and $175^{\circ}(\nabla)$. The solid curves represent the theoretical values for the ideal chains calculated from eqs 14 and 15 . The upper and lower dotted horizontal lines represent the asymptotic values $7 / 9$ in the ideal (unperturbed) random-coil limit ${ }^{8}$ and 4/9 in the rigid-rod limit, ${ }^{7}$ respectively.

In the case of $\theta=109^{\circ}$ (typical flexible chain), the MC value 0.76 of $g_{S}$ for $n=900$ is somewhat (ca. 2\%) smaller than the value $7 / 9(=0.778)$ in the ideal random-coil limit. The available experimental values of $g_{S}$ for regular $f$-arm star polystyrenes (PS) in good solvents obtained by Khasat et al. ${ }^{23}$ for $f=3$ and by Okumoto et al. ${ }^{24,25}$ for $f=4$ and 6 are 0.79 , 0.61 , and 0.45 , respectively, and are in good agreement with the respective ideal random-coil limiting values $7 / 9(=0.778)$, $5 / 8(=0.625)$, and 4/9 $(=0.444)$, which have been calculated from $g_{S}=(3 f-2) / f^{2} .8$ The available theoretical values of $g_{S}$ obtained by Douglas and Freed ${ }^{9}$ on the bases of the polymer RG theory for the regular three-, four-, and six-arm stars in the perturbed random-coil limit are 0.778, 0.631, and 0.453, respectively, and are consistent with the experimental ones. Then the present $\mathrm{MC}$ result for long flexible chains is consistent with the above-mentioned experimental and theoretical results.

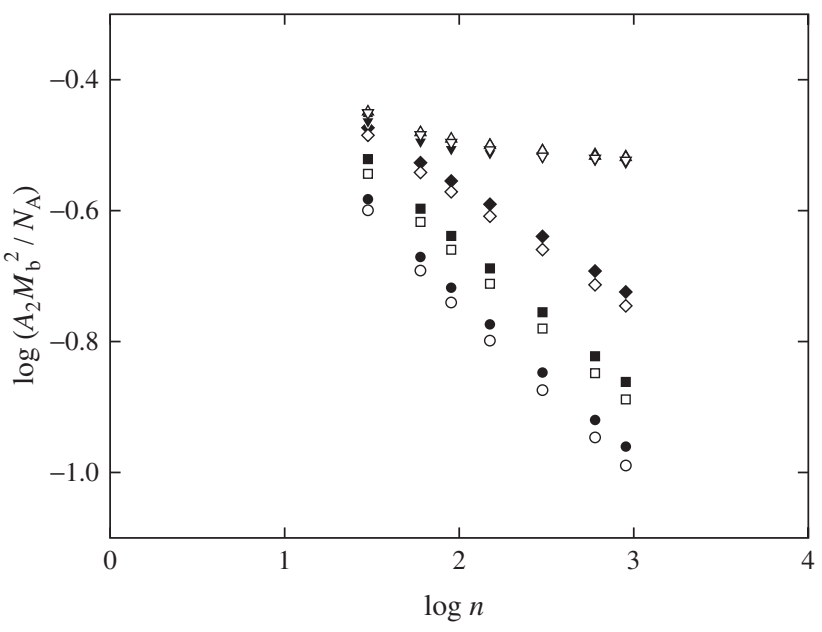

Figure 3. Double-logarithmic plots of $A_{2} M_{\mathrm{b}}{ }^{2} / N_{\mathrm{A}}$ against $n$ at $T^{*}=8.0(\mathrm{good}$ solvent system). All the symbols have the same meaning as those in Figure 1.

As a natural consequence of the results shown in Figure 1, the MC value of $g_{S}$ becomes almost identical with the ideal chain value as the chain becomes stiffer. The ratio $g_{S}$, on the whole, remarkably depends on the chain stiffness but scarcely on the intramolecular excluded volume.

\section{Second Virial Coefficient}

We have numerically evaluated $A_{2} M^{2} / N_{\mathrm{A}}$ from eqs 11-13 on the basis of the sets of sample configurations generated in the above-mentioned MC evaluation of $\left\langle S^{2}\right\rangle$. The MC values of $A_{2} M_{\mathrm{b}}{ }^{2} / N_{\mathrm{A}}$ with $M_{\mathrm{b}}=M / n$ so obtained for the star and linear chains are given in the third and fifth columns, respectively, of Table I, the number in the parentheses attached to each value indicating its statistical error. The $A_{2} M_{\mathrm{b}}{ }^{2} / N_{\mathrm{A}}$ value and its error for each chain are the mean and the standard deviation, respectively, of independent $\mathrm{MC}$ results.

Figure 3 shows double-logarithmic plots of $A_{2} M_{\mathrm{b}}{ }^{2} / N_{\mathrm{A}}$ against $n$ at $T^{*}=8.0$ (good solvent system), where all the open and closed symbols have the same meaning as those in Figure 1 . In the case of $\theta=109^{\circ}$ (typical flexible chain), the value for the regular three-arm star is somewhat (6\% at most) smaller than the corresponding value for the linear chain. For both the star and linear chains, $A_{2}$ decreases with increasing $n$ and the slopes of the plots become almost identical with the asymptotic value -0.2 for very large $n$. The difference in the value of $A_{2}$ between the two chains becomes small and the slopes become gentle, as $\theta$ (chain stiffness) is increased. In the case of $\theta=175^{\circ}$ (typical semiflexible or stiff chain), $A_{2}$ becomes almost independent of $n$ for $n \geq 100$, as in the case of the rigid rod.

It is interesting to note that the value of $A_{2}$ for the chain of $\theta=175^{\circ}$ is smaller than that of $\theta=165^{\circ}$ over the whole range of $n$ examined. It may be regarded as arising from the fact that two chains with the LJ potential (having the attractive interaction), which are close to each other, prefer to be parallel rather than perpendicular to each other when their stiffness becomes large and their $A_{2}$ may therefore be suppressed, ${ }^{26}$ as 


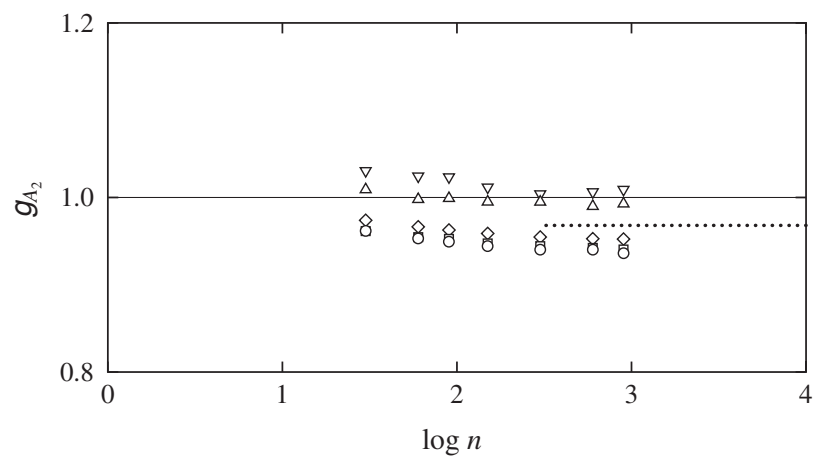

Figure 4. Plots of $g_{A_{2}}$ against $\log n$ at $T^{*}=8.0$ (good solvent system). All the symbols have the same meaning as those in Figure 2. The dotted horizontal line segment represents the RG theory value ${ }^{9}$ 0.968 .

discussed by Schoot and Odijk ${ }^{27}$ in the case of long rigid rods with a van der Waals-type potential.

Figure 4 shows plots of $g_{A_{2}}$ against $\log n$ at $T^{*}=8.0(\operatorname{good}$ solvent system). The symbols, which have the same meaning as those in Figure 2, represent the MC values of $g_{A_{2}}$ calculated from the values of $A_{2} M_{\mathrm{b}}{ }^{2} / N_{\mathrm{A}}$ given in the third and fifth columns in Table I. We note that from the defining eq 1 for the effective intermolecular excluded volume $V_{\mathrm{E}}, g_{A_{2}}$ may be written in terms of $V_{\mathrm{E}}$ as follows,

$$
g_{A_{2}}=\frac{A_{2}(\text { star })}{A_{2}(\text { linear })}=\frac{V_{\mathrm{E}}(\text { star })}{V_{\mathrm{E}}(\text { linear })}
$$

In the case of $\theta=109^{\circ}$ (typical flexible chain), $g_{A_{2}}$ slightly decreases with increasing $n$ and then seems to approach a constant independent of $n$. The asymptotic value of $g_{A_{2}}$ in the limit of $n \rightarrow \infty$ may be estimated to be 0.94 which is the mean of the three MC values $0.94_{0}, 0.94_{0}$, and $0.93_{6}$ for $n=300$, 600 , and 900 , respectively. The $g_{A_{2}}$ value so obtained is rather in good agreement with the RG theory value ${ }^{9} 0.968$ represented by the dotted horizontal line segment in Figure 4 . In this connection, we refer to literature experimental and theoretical values of $g_{A_{2}}$ for regular stars. The former values are 0.89 and 0.79 for the regular four- and six-arm star PSs,${ }^{24,25}$ respectively, and they are rather in good agreement with the corresponding RG theory values ${ }^{9} 0.923$ and 0.808 . Strictly speaking, the RG theory values are somewhat (ca. 3\%) larger than the corresponding experimental and present MC values.

It is more important to see in Figure 4 that $g_{A_{2}}$ slightly increases with increasing $\theta$ (chain stiffness) in the range of $\theta$ from $109^{\circ}$ to $165^{\circ}$ and it becomes almost identical with unity for $\theta=165^{\circ}$ and $175^{\circ}$ in the range of $n \gtrsim 100$. We note that $g_{A_{2}}$ in the cases of $\theta=165^{\circ}$ and $175^{\circ}$ approaches, of course, the above-mentioned asymptotic value in the limit of $n \rightarrow \infty$. In contrast to the cases of $g_{S}$ and $g_{\eta}, g_{A_{2}}$ is rather insensitive to change in $\theta$ (chain stiffness). Such characteristic behavior of $g_{A_{2}}$ may be regarded as arising from the fact that in terms of the (classical) perturbation theory, ${ }^{4}$ effects of the multiple intermolecular contact become negligibly small, if any, in the rigidrod limit. The decreases in $A_{2}$ of the star and linear chains

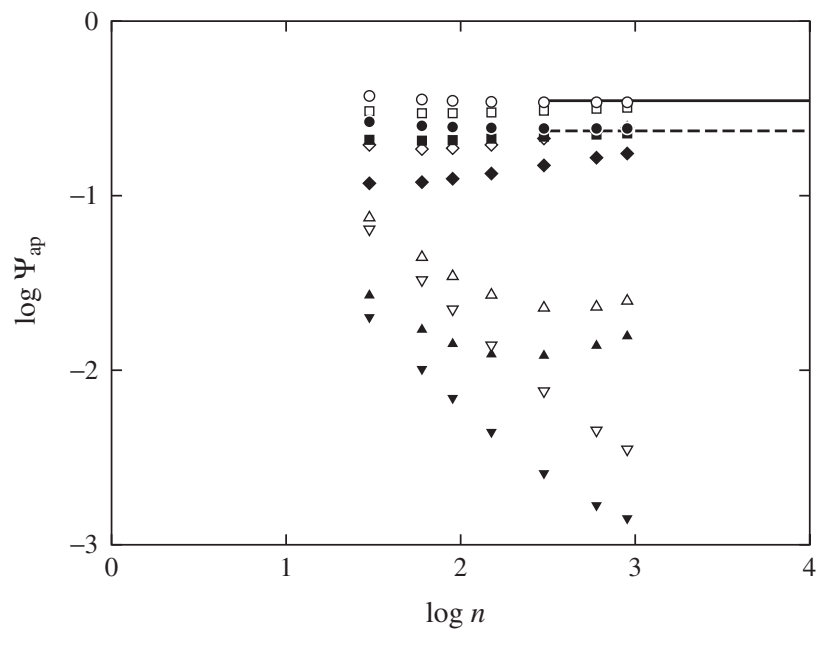

Figure 5. Double-logarithmic plots of $\Psi_{\text {ap }}$ against $n$ at $T^{*}=8.0$ (good solvent system). All the symbols have the same meaning as those in Figure 1. The solid horizontal line segment represents the asymptotic value 0.35 in the random-coil limit obtained by Ohno et al. ${ }^{31}$ for the self-avoiding regular three-arm star chain on the simple cubic lattice and the dashed one the corresponding value 0.235 obtained by Barrett ${ }^{32}$ for the self-avoiding linear chain on the same lattice.

due to the attractive interaction in the LJ potential may be considered to cancel out with each other in $g_{A_{2}}$.

Finally, we consider the apparent interpenetration function $\Psi_{\text {ap }}$ defined by ${ }^{28}$

$$
A_{2}=4 \pi^{3 / 2} N_{\mathrm{A}} \frac{\left\langle S^{2}\right\rangle^{3 / 2}}{M^{2}} \Psi_{\text {ap }}
$$

from the whole $A_{2}$ including effects of chain ends. ${ }^{29,30}$ Figure 5 shows double-logarithmic plots of $\Psi_{\text {ap }}$ against $n$ at $T^{*}=8.0$ (good solvent system). The open and closed symbols, which have the same meaning as those in Figures 1 and 3, represent the MC values for the star and linear chains, respectively, calculated from eq 17 with the values of $\left\langle S^{2}\right\rangle / n$ and $A_{2} M_{\mathrm{b}}{ }^{2} / N_{\mathrm{A}}$ given in Table I.

In the case of $\theta=109^{\circ}$ (typical flexible chain), $\Psi_{\text {ap }}$ for both the star and linear chains slightly decreases with increasing $n$ and then seems to approach the asymptotic values 0.34 and 0.24 , respectively, which are the means of the three $\Psi_{\text {ap }}$ values for $n=300,600$, and 900, although $\Psi_{\text {ap }}$ is appreciably larger for the star chain than for the linear one. The two asymptotic values are in good agreement with the literature MC values 0.35 for the star chain and 0.235 for the linear one obtained by Ohno et al. $^{31}$ and Barrett, ${ }^{32}$ respectively, by the use of selfavoiding chains on the simple cubic lattice. In Figure 5, the two literature values for the star and linear chains are represented by the solid and dashed horizontal line segments, respectively. We note that the RG theory values ${ }^{9} 0.384$ and 0.269 of $\Psi_{\text {ap }}$ for the perturbed flexible regular three-arm star and linear chains, respectively, are somewhat (ca. 10\%) larger than the above-mentioned present and literature MC values. As $\theta$ (chain stiffness) is increased from $109^{\circ}$ to $175^{\circ}, \Psi_{\text {ap }}$ decreases monotonically. 


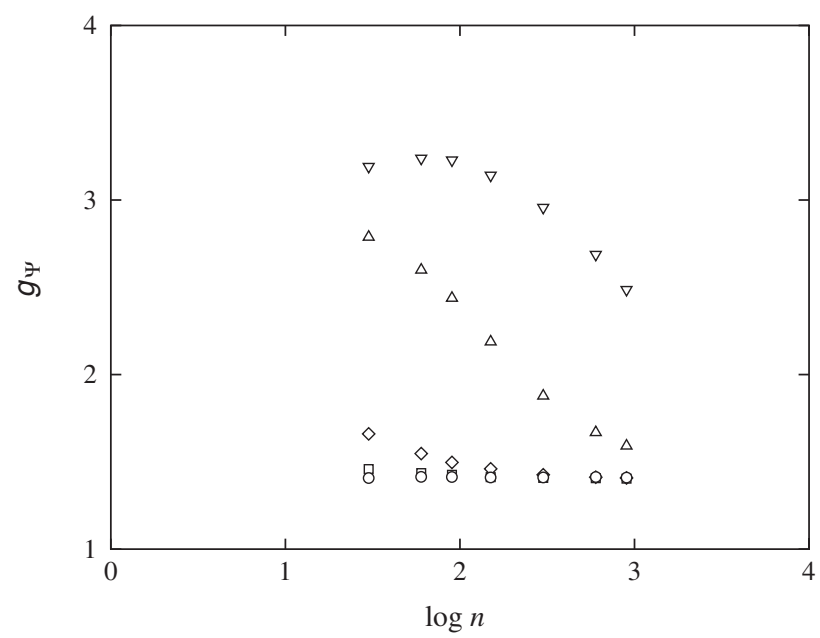

Figure 6. Plots of $g_{\Psi}$ against $\log n$ at $T^{*}=8.0($ good solvent system). All the symbols have the same meaning as those in Figure 2.

From eqs 16 and $17, g_{A_{2}}$ may be written in the form,

$$
g_{A_{2}}=g_{S}^{3 / 2} g_{\Psi}
$$

where $g_{\Psi}$ is the ratio of $\Psi_{\text {ap }}$ of the regular three-arm star chain to that of the corresponding linear one. Figure 6 shows plots of $g_{\Psi}$ against $\log n$ at $T^{*}=8.0($ good solvent system), where all the symbols have the same meaning as those in Figures 2 and 4 . It is seen that $g_{\Psi}$ remarkably increases with increasing $\theta$ (chain stiffness). From eq 18 with the results shown in Figures 2, 4, and 6, it may be said that the increase in $g_{\Psi}$ compensates the decrease in $g_{S}{ }^{3 / 2}$ and therefore $g_{A_{2}}$ is insensitive to change in $\theta$ (chain stiffness).

\section{CONCLUSION}

We have examined the effects of chain stiffness on the ratio $g_{A_{2}}$ of $A_{2}$ of the regular three-arm star chain to that of the corresponding linear one on the basis of the MC results for $A_{2}$ and $\left\langle S^{2}\right\rangle$ of the freely rotating chains with the LJ 6-12 potential corresponding to a good solvent system. It is found that $g_{A_{2}}$ is rather insensitive to change in chain stiffness in contrast to the cases of the ratios $g_{S}$ and $g_{\eta}$ related to $\left\langle S^{2}\right\rangle$ and $[\eta]$, respectively, which remarkably decrease with increasing chain stiffness. Such characteristic behavior of $g_{A_{2}}$ may be regarded as arising from the fact that in terms of the (classical) perturbation theory, ${ }^{4}$ the effects of the multiple intermolecular contact become negligibly small, if any, in the rigid-rod limit, or in other words, the increase in the ratio $g_{\Psi}$ related to $\Psi_{\text {ap }}$ compensates the decrease in $g_{S}{ }^{3 / 2}$.

Received: July 17, 2008

Accepted: July 29, 2008

Published: September 11, 2008

\section{REFERENCES AND NOTES}

1. D. Ida and T. Yoshizaki, Polym. J., 39, 1373 (2007).

2. D. Ida, Y. Nakamura, and T. Yoshizaki, Polym. J., 40, 256 (2008).

3. J. G. Kirkwood and J. Riseman, J. Chem. Phys., 16, 565 (1948).

4. H. Yamakawa, "Modern Theory of Polymer Solutions," Harper \& Row, New York, 1971. Its electronic edition is available on-line at the URL: http://www.molsci.polym.kyoto-u.ac.jp/archives/redbook.pdf

5. B. H. Zimm and R. W. Kilb, J. Polym. Sci., 37, 19 (1959).

6. I. M. Irurzun, J. Polym. Sci., Part B: Polym. Phys., 35, 563 (1997).

7. M. L. Mansfield and W. H. Stockmayer, Macromolecules, 13, 1713 (1980).

8. B. H. Zimm and W. H. Stockmayer, J. Chem. Phys., 17, 1301 (1949).

9. J. F. Douglas and K. F. Freed, Macromolecules, 17, 1854 (1984).

10. W. G. McMillan and J. E. Mayer, J. Chem. Phys., 13, 276 (1945).

11. J. P. Hansen and I. R. McDonald, "Theory of Simple Liquids," 3rd ed., Academic, London, 2006.

12. H. Yamakawa and T. Yoshizaki, J. Chem. Phys., 118, 2911 (2003).

13. H. Yamakawa and T. Yoshizaki, J. Chem. Phys., 119, 1257 (2003).

14. M. Lal, Mol. Phys., 17, 57 (1969).

15. N. Madras and A. D. Sokal, J. Stat. Phys., 50, 109 (1988).

16. N. Metropolis, A. W. Rosenbluth, M. N. Rosenbluth, A. H. Teller, and E. Teller, J. Chem. Phys., 21, 1087 (1953).

17. S. D. Stellman and P. J. Gans, Macromolecules, 5, 516 (1972).

18. S. D. Stellman, M. Froimowitz, and P. J. Gans, J. Comput. Phys., 7, 178 (1971).

19. M. Matsumoto and T. Nishimura, ACM Trans. Model. Comput. Simul., 8, 3 (1998), see also the URL: http://www.math.keio.ac.jp/ matsumoto/emt.html

20. M. Guenza, M. Mormino, and P. Perico, Macromolecules, 24, 6168 (1991).

21. W. Bruns, Macromolecules, 17, 2826 (1984).

22. H. Yamakawa and T. Yoshizaki, J. Chem. Phys., 121, 3295 (2004).

23. N. Khasat, R. W. Pennisi, N. Hadjichristidis, and L. J. Fetters, Macromolecules, 21, 1100 (1988).

24. M. Okumoto, Y. Nakamura, T. Norisuye, and A. Teramoto, Macromolecules, 31, 1615 (1998).

25. M. Okumoto, Y. Iwamoto, Y. Nakamura, and T. Norisuye, Polym. J., 32, 422 (2000).

26. D. Ida and T. Yoshizaki, J. Chem. Phys., in press.

27. P. van der Schoot and T. Odijk, J. Chem. Phys., 97, 515 (1992).

28. Y. Einaga, F. Abe, and H. Yamakawa, Macromolecules, 26, 6243 (1993).

29. H. Yamakawa, Macromolecules, 25, 1912 (1992).

30. H. Yamakawa, "Helical Wormlike Chains in Polymer Solutions," Springer, Berlin, 1997.

31. K. Ohno, K. Shida, M. Kimura, and Y. Kawazoe, Macromolecules, 29, 2269 (1996).

32. A. J. Barrett, Macromolecules, 18, 196 (1985). 